\title{
Extragastrointestinal stromal tumor originating from the vulva
}

\author{
TAKESHI FUKUDA, TOSHIYUKI SUMI, YUSUKE NAKANO, MASANARI MORISHITA, HIROYUKI NOBEYAMA, \\ HIROYUKI YOSHIDA, YOSHINARI MATSUMOTO, TOMOYO YASUI, KEN-ICHI HONDA and OSAMU ISHIKO
}

Department of Obstetrics and Gynecology, Osaka City University Graduate School of Medicine, Osaka 545-8585, Japan

Received January 11, 2011; Accepted June 22, 2011

DOI: $10.3892 / \mathrm{ol} .2011 .343$

\begin{abstract}
Gastrointestinal stromal tumors (GISTs) are the most common mesenchymal tumors of the gastrointestinal tract. They have gain-of-function mutations of the c-kit receptor tyrosine kinase gene and have been suggested to originate from the interstitial cells of Cajal. A small percentage of GISTs form extragastrointestinal masses. We report a rare case of a GIST originating from the vulva. A 55-year-old woman presented with a vulvar tumor. The tumor was initially diagnosed as a leiomyosarcoma following the first resection. Following a second recurrence the patient was administered chemotherapy. A third recurrence occurred and the patient underwent a third resection. Histology revealed that a bundle of fibrous tumor cells had invaded the connected tissue and muscular coat, and some spindle-shaped and blunt-ended nuclei were detected. Furthermore, immunohistochemical evaluation revealed that the tumor cells exhibited strong and diffuse staining for c-kit and CD34. The recurrent tumor was diagnosed as a GIST and a reevaluation of the original specimens also revealed a GIST. The patient was treated with imatinib, and is currently healthy with no evidence of recurrence at 20 months after the last surgery.
\end{abstract}

\section{Introduction}

Gastrointestinal stromal tumors (GISTs) are the most common mesenchymal tumors of the gastrointestinal tract, with approximately $70 \%$ of the tumors occurring in the stomach, $20-30 \%$ in the small intestine and less than $10 \%$ in the esophagus, colon and rectum (1). The most practical and specific diagnostic criterion for GIST is immunohistochemically determined c-kit (CD117) expression (2). A GIST is considered to be a particular type of tumor that originates from an interstitial cell of Cajal (ICC) or its precursor $(2,3)$. ICCs are the pacemakers of the peristaltic movement of the gastrointestinal tract (4). A small percentage of GISTs form extragastrointestinal masses in the

Correspondence to: Dr Toshiyuki Sumi, Department of Obstetrics and Gynecology, Osaka City University Graduate School of Medicine, 1-4-3, Asahimachi, Abeno-ku, Osaka 545-8585, Japan E-mail:sumi-toshi@med.osaka-cu.ac.jp

Key words: gastrointestinal stromal tumor, vulva, leiomyosarcoma, imatinib omentum, mesenteries, retroperitoneum, rectovaginal septum and undefined abdominal sites. These tumors are occasionally designated as extragastrointestinal stromal tumors (5).

We report a rare case of a GIST originating from the vulva, which was initially diagnosed as a leiomyosarcoma and correctly diagnosed following a second recurrence.

\section{Case report}

A 55-year-old woman presented with vulvar swelling and pain. She was found to have a tumor that was larger than a hen's egg with a multilobular surface in the left vulva. She underwent resection of the tumor and the tumor was pathologically diagnosed as a leiomyosarcoma. The patient was then referred to our hospital for further examination and treatment for leiomyosarcoma. Pelvic examination revealed no residual tumor in the vulva, and results of a biopsy also indicated no tumor. The specimen from the former hospital was reexamined and confirmed to be a leiomyosarcoma. The patient underwent a simple vulvectomy and biopsy of the superficial inguinal lymph nodes. The pathologic specimen was interpreted to be a leiomyosarcoma (Fig. 1), with no metastatic tumors evident in the lymph nodes. Subsequently, she underwent adjuvant radiation, receiving $60 \mathrm{~Gy}$ to the pelvis.

After 2 years and 4 months, the patient presented with vulvar pain and was found to have a $1.5-\mathrm{cm}$ recurrent mass in the left vulva. After a second resection, the histological diagnosis was a leiomyosarcoma. The patient underwent three chemotherapy cycles with irinotecan (CPT-11) and cisplatin (CDDP), comprising CDDP at $60 \mathrm{mg} / \mathrm{m}^{2}$ body surface area (BSA) on day 1 plus CPT-11 at $60 \mathrm{mg} / \mathrm{m}^{2}$ BSA on days 1,8 and 15 .

After another 2 years, she presented with vulvar pain and was found to have a palpable $3-\mathrm{cm}$ recurrent mass in the left vulva, which was detected by pelvic MRI (Fig. 2), and she underwent a third resection. Histology revealed that a bundle of fibrous tumor cells had invaded the connected tissue and muscular coat, and that there were some spindle-shaped and blunt-ended nuclei present. On immunohistochemical evaluation, the tumor cells exhibited strong and diffuse staining for c-kit and CD34, with no staining for desmin or $\alpha$-smooth muscle actin (Fig. 3). This recurrent tumor was diagnosed as a GIST and reevaluation of the original specimens also revealed a GIST (Fig. 4). The patient was treated with imatinib at $400 \mathrm{mg} /$ day. On day 13, she presented with systemic exanthema and immediately stopped taking imatinib. Later she restarted 

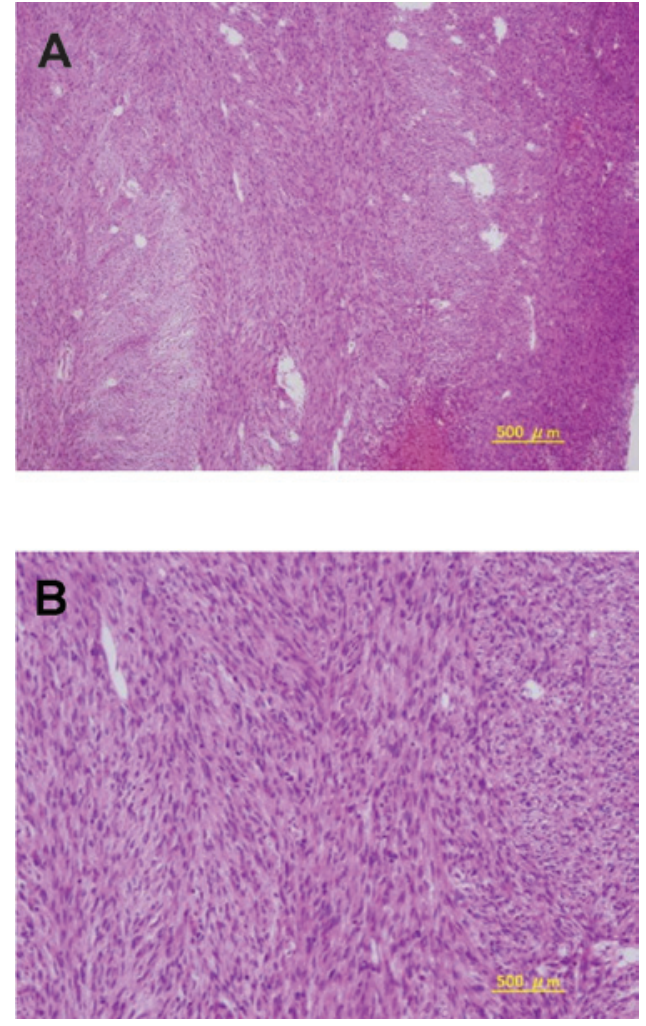

Figure 1. Spindle cells with high cell density and mitotic activity. (A) Low-power view; (B) high-power view.

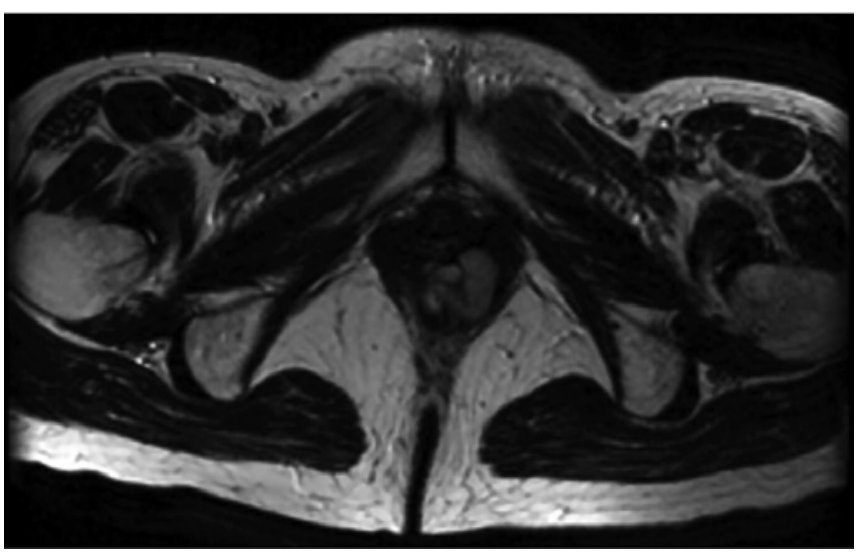

Figure 2. T2-weighted MR image showing a high-intensity mass $(3 \mathrm{~cm})$ in the left vulva.

imatinib at a lower dose (100 $\mathrm{mg} /$ day) with administration of $\mathrm{H} 1$ antihistamine. No erythematous lesions developed, and the dose of imatinib was subsequently increased to $400 \mathrm{mg} /$ day. The patient is currently healthy with no evidence of recurrence at 20 months after the last surgery.

\section{Discussion}

GISTs are the most common mesenchymal tumors of the gastrointestinal tract. They have gain-of-function mutations of the c-kit receptor tyrosine kinase gene and have been suggested to originate from ICCs (3). A small percentage of GISTs arise from extragastrointestinal masses in the omentum, mesenteries, retroperitoneum, rectovaginal septum and
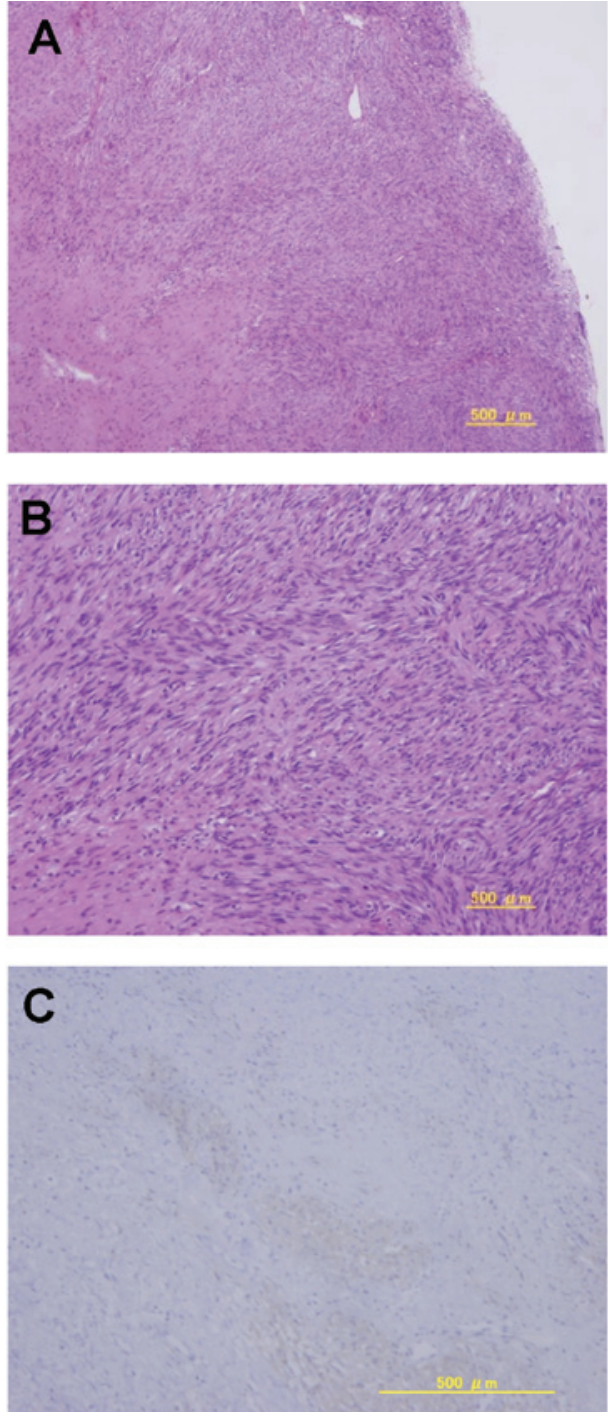

Figure 3. (A and B) Spindle cells with high cell density and mitotic activity; (A) low-power view, (B) high-power view. (C) The lesion cells are diffusely positive for c-kit.

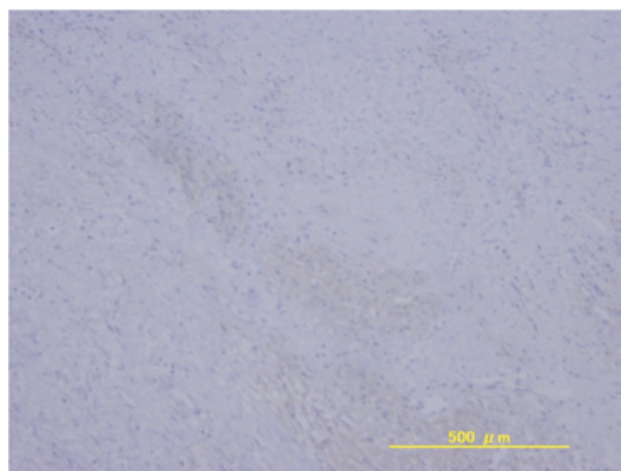

Figure 4. The lesion cells are diffusely positive for c-kit.

undefined abdominal sites. These tumors are occasionally designated as extragastrointestinal stromal tumors (5). Miettinen et al (6) reported that extragastrointestinal stromal tumors may not arise from ICCs, but instead may be multipotential neoplasms that exhibit divergent differentiation (Cajal cell-like or smooth muscle cell-like). The accurate rate 
of GIST occurrence is unclear. Miettinen et al (7) reported that GISTs most commonly occur in the stomach $(60 \%)$, jejunum/ileum (30\%), duodenum (4-5\%), rectum (4\%) and colon/appendix (1-2\%). The prognosis of GISTs, including extragastrointestinal stromal tumors, is predicted by their size and mitotic rate. Consensus guidelines were issued to stratify GISTs by their size and mitotic rate into four categories according to the risks of aggressive behavior, i.e., very low, low, intermediate and high risk (8). Complete excision should be performed whenever possible during treatment of a GIST. If the tumor is surgically incurable, adjuvant imatinib treatment has been approved (9). Most adverse reactions to imatinib are mild to moderate and include nausea, diarrhea, myalgia, edema and cutaneous reactions. Approximately $31-44 \%$ of patients taking imatinib experience cutaneous reactions (10). A rash is more likely to occur with higher doses and may therefore be a pharmacological effect rather than an immunogenic or hypersensitivity reaction. The rash generally responds to steroids, antihistamines and emollients. In addition, a gradual increase in the dose can reinstate imatinib therapy following resolution of the cutaneous eruptions (11). In the present case, we were able to reinstate imatinib therapy at a lower dose with an antihistamine, and the patient is currently healthy with no evidence of recurrence at 20 months after the last surgery.

In conclusion, we have reported a rare case of a GIST that presented as a vaginal tumor. Gynecologists should be aware that GISTs may occur in this location, since a correct diagnosis is crucial for providing information and initiating imatinib therapy.

\section{References}

1. Miettinen M, Sarlomo-Rikala M and Lasota J: Gastrointestinal stromal tumors: recent advances in understanding of their biology. Hum Pathol 30: 1213-1220, 1990.
2. Hirota S, Isozaki K, Moriyama Y, Hashimoto K, Nishida T, Ishiguro S, Kawano K, Hanada M, Kurata A, Takeda M, Muhammad Tunio G, Matsuzawa Y, Kanakura K, Shinomura Y and Kitamura Y: Gain-of-function mutations of c-kit in human gastrointestinal stromal tumors. Science 279: 577-580, 1998.

3. Kindblom LG, Remotti HE, Aldenborg F and Meis-Kindblom JM: Gastrointestinal pacemaker cell tumor (GIPACT): gastrointestinal stromal tumors show phenotypic characteristics of the interstitial cell of Cajal. Am J Surg Pathol 152: 1259-1269, 1998.

4. Thomsen L, Robinson TL, Lee JC, Farraway LA, Hughes MJ, Andrews DW and Huizinga JD: Interstitial cells of Cajal generate a rhythmic pacemaker current. Nat Med 4: 848-851, 1998.

5. Miettinen M, Sobin LH and Lasota J: Gastrointestinal stromal tumors presenting as omental masses - a clinicopathologic analysis of 95 cases. Am J Surg Pathol 33: 1267-1275, 2009.

6. Miettinen M, Monihan JM, Sarlomo-Rikala M, Kovatich AJ, Carr NJ, Emory TS and Sobin LH: Gastrointestinal stromal tumors/smooth muscle tumors (GISTs) primary in the omentum and mesentery: clinicopathologic and immunohistochemical study of 26 cases. Am J Surg Pathol 23: 1109-1118, 1999.

7. Miettinen $\mathrm{M}$ and Lasota $\mathrm{J}$ : Gastrointestinal stromal tumors: pathology and prognosis at different sites. Semin Diagn Pathol 23: 70-83, 2006.

8. Fletcher CD, Berman JJ, Corless C, et al: Diagnosis of gastrointestinal stromal tumors: a consensus approach. Hum Pathol 33 459-465, 2002.

9. Nilsson B, Sjölund K, Kindblom LG, Meis-Kindblom JM, Bümming P, Nilsson O, Andersson J and Ahlman H: Adjuvant imatinib treatment improves recurrence-free survival in patients with high-risk gastrointestinal stromal tumors (GIST). Br J Cancer 96: 1656-1658, 2007.

10. Deininger MW, O'Brien SG, Ford JM and Druker BJ: Practical management of patients with chronic myeloid leukemia receiving imatinib. J Clin Oncol 21: 1637-1647, 2003.

11. Scott LC, White JD, Reid R and Cowie F: Management of skin toxicity related to the use of imatinib mesylate (STI571, Glivec ${ }^{\mathrm{TM}}$ ) for advanced stage gastrointestinal stromal tumors. Sarcoma 9: 157-160, 2005. 